\section{Bioinformation}

www.bioinformation.net

\title{
CDAC - Development of a curated database of anticancer compounds from marine resources
}

\author{
Sundaramoorthy Hari Prasad ${ }^{1 *}$ and Munuswamy Deccaraman ${ }^{2}$ \\ ${ }^{1}$ Department of Bioinformatics and Biotechnology, Vinayaga Mission University, Salem, India; ${ }^{2}$ Department of Industrial Biotechnology, \\ Dr. MGR University, Maduravoyal, Chennai, India 600095; \\ Sundaramoorthy Hari Prasad - E-mail: hpbiochem@yahoo.co.in; * Corresponding author
}

received June 24, 2007; revised June 30, 2007; accepted July 19, 2007; published online December 01, 2007

\begin{abstract}
:
Anticancer compounds from marine source find application in cancer treatment. Several such compounds have been identified and documented. Here, we describe the development of CDAC, a curated database on anticancer compounds from marine sources.
\end{abstract}

Keywords: anticancer; drugs; curated database; marine

\section{Background:}

The marine source is a rich reserve for many important small molecule compounds. [1] It serves as a diversity pool for anticancer compounds. Numerous drugs from marine sources are traditionally used for treating ailments. In modern drug discovery, many compounds identified to have anticancer activity do not enter the pharmaceutical market due to competition and stringent Food and Drug Administration (FDA) policies. [2, 3] However, computational analyses have accelerated the discovery process. Such analysis can be further improved using data collected on compounds from different natural sources in the form of a comprehensive database. A database of anticancer compounds from marine sources is not available. Here, we describe the development of a database containing data on anticancer compounds from marine sources.

\section{Database Model:}

Data mining

Published articles containing data on anticancer compounds from marine sources were collected from PUBMED [6] and PUBMED CENTRAL [7] using KEYWORD search. Data on molecular weight, molecular formula and SMILES notation are retrieved from PUBCHEM.

\section{Database development}

The server used in this application is Apache Tomcat and the database is MySQL. A web based application is developed for the database using JSP (Java Server Pages). The demo database is made available for free at http://demo.cheapchess.com/hpcancer/

\section{Future development:}

The manual mining process is laborious, time consuming and error prone. The future plan is to replace manual creation by automated methods.

\section{References:}

[01] J. M. Arif, et al., J Biomed Biotechnol., 93 (2004)

[02] M. L. Amador, et al., Annals of Oncology, 14: 1607 (2003)

[03] L. Simmons, et al., Mol Cancer Ther., 4 (2005)

[04] P. Proksch, et al., Appl Microbiol Biotechnol., 59: 125 (2002)

[05] L. F. Garcia-Fernandez, et al., Pharmaceut News, 9: 495 (2002)

[06] http://www.ncbi.nlm.nih.gov/entrez/query.fcgi?d $\mathrm{b}=$ pubmed

[07] http://www.ncbi.nlm.nih.gov/entrez/query.fcgi?d $\mathrm{b}=\mathrm{PMC}$

Edited by $P$. Kangueane

Citation: Prasad \& Deccaraman, Bioinformation 2(3): 96 (2007)

License statement: This is an open-access article, which permits unrestricted use, distribution, and reproduction in any medium, for non-commercial purposes, provided the original author and source are credited. 